\title{
Research on Land Warfare Simulation Framework Based on Multi-Agent
}

\author{
Gan Bin and Hao Jiaxin
}

\begin{abstract}
Networking-centric land warfare simulation is an evolving complex adaptive system that represents the superior trend of the land warfare demonstration. As a new complex combat system of systems, Agent-based modeling and simulation (ABMS) proves an effective approach to explore the new operational characteristics. In this paper, we discuss the background to the agent-based modeling and simulation and how it work, and then we use eight indicators to model the different types of the land warfare agent and discuss the engagement rules for the land warfare agent. We also present a discussion of the simulation framework based on multi-agent. A prototype system MABLWS (Multi-agent based land warfare simulation) was accomplished on the basis of methods before-mentioned. The simulation results based on a typical scenario show that the agent-based method would have favorable future in the field of research on land warfare simulation.
\end{abstract}

Index Terms-Land warfare, multi-agent, simulation framework, network-centric warfare.

\section{INTRODUCTION}

Land warfare simulation system is an evolving complex adaptive system [1], [2]. Land warfare simulation system consists of a large number of elements (known as all levels of combat units) where there are numerous non-linear interactions between these elements. These combat units constitute a hierarchy (command and control structure) of the system, where the fractional chaotic behavior of the lowlevel elements would prompt the orderly pattern of behavior remained in the high-level. The component units of the land warfare must adapt to the changing battlefield environment in order to surviving. Warfare is always asymmetric and far from equilibrium. Based on network-centric warfare pattern, there is no centralized command and control organization which is responsible for the direct guidance of commanding the specific actions for each unit. Obviously, land warfare possesses the main features of the complex adaptive systems, such as non-linear, hierarchical, self- organized, adaptive, unbalanced and decentralized control. These natures cannot be described by the traditional model of warfare (e.g. Lanchester Equation).

Currently, Agent-based modeling and simulation (ABMS) proves an effective approach to explore the new operational characteristics of the complex system. The paper briefly discusses the background to the agent-based modeling and simulation and how it work, followed by two sections that detail the requirements for the agent model and discuss the

Manuscript received August 22, 2013; revised October 29, 2013

The authors are with Science and Technology on Complex Systems Simulation Laboratory, Beijing, China (email: colebin@aliyun.com, haojiaxin@126.com). agent-based framework design

\section{Multi-Agent Based Modeling AND Simulation}

Multi-agent based system is referred to the dynamic environment system composed of many autonomous or semi-autonomous objects which influence on or interact with each other, and perform some specific tasks. The multiagent based system includes the environment; the entities (property and state) in the physical environment as well as the rules followed by the physical entity which interacting with the others or the physical environment, showed in Fig. 1. Multi-Agent modeling and simulation technology is focused on the study of the viable entity in the system, that be called as Agent. Compared with the general object, Agent is a more intelligent and autonomous individual, which can sense the changes in the external environment, response to the external stimuli and take more adaptive action selection according to the internal rules in order to survive in the environment. By modeling and simulating the agents in the complex system and the interactions between the agents, the micro-level behavior and the macro-level phenomena ("Emergence") of the complex system can be organically combined. Multi-agent modeling and simulation is a topdown analysis, bottom-up integrated modeling approach.

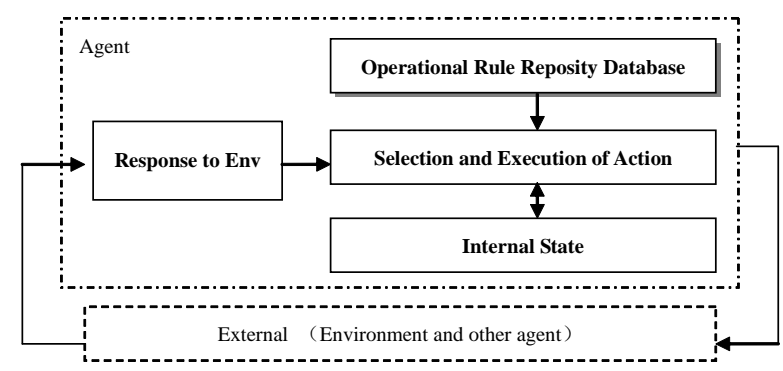

Fig. 1. Diagram of Multi-agent based system.

Multi-Agent-based modeling and simulation is focused on the behavioral layer, and explore the tendency to physical behavior of the system according to the simple action of the underlying individuals, as followed as [3]:

- By specifying the internal state changes in the intelligent individuals and observing the massive results of interaction between the agents, trying to find the phenomena of emergence of complex systems, this is a bottom-up modeling and simulation method;

- In the multi-agent based modeling and simulation, there is no central coordinating agent, that is, there is no centralized control;

- Assume usually, the individual agent uses relatively simple rules to interact with other agents or the environment. 


\section{Multi-Agent Based LAND WARFARE Simulation MODELS}

\section{A. Modeling for the Combat Individual}

Firstly, multi-agent based land warfare modeling is required to determine the main features of the agent in the operational process, which can be used to distinguish the different types of combat units. By analyzing and summarizing the internal characteristic of the army force, MABLWS (agent-based land warfare simulation) uses the following main eight indicators to model the different types of the combat individual.

1) The ability to move. This is the main characteristics of the combat agent, which can be expressed by the dynamics algorithm of the entity according to the features of terrain or by the maximum movement range of the entity in each simulation step. Based on trade-offs, MABLWS uses the maximum speed on different types of terrain as the ability to move for the individuals.

2) The sensing range. The combat individual must have the ability to sense other agents and the environmental state, which is the key ability for interacting. The sensing ability of the combat agent includes optical and electronic reconnaissance capabilities, which can be influenced by many factors, such as the distance of the target, the environment (smoke, atmospheric attenuation factor), LOS, etc. To simplify the problem, MABLWS uses the maximum sensing distance as the sensing ability for the individuals

3) The ability of communication. As the sensing range, the communication is the key to the interaction of the agents However, the ability of communication does not mean the perception of the individuals, which is used to exchange information between agents far from the sensing range using the communication equipments.

4) Lethality. The destruction degree of the weapons carried by the combat individuals is described by the Lethality, which reflects the different capacity of weapon to fight against the enemy.

5) The effective range. The effective range represents the maximum range of the different weapons carried by the individuals.

6) Viability. Viability is an indicator to distinguish different types of individuals, e.g. the viability of soldiers and tanks is different. MABLWS defines a number between $[0,1]$ as the viability for the individuals.

7) Probability of hit. The probability of hit is used to express the hit probability of the target during the engagement.

8) The weights for attributes. The attribute weights of the individuals reflect the individual's operational intent, selfprotection capabilities, loyalty and obedience.

\section{B. Modeling for the Engagement Rules}

Doctrines are theoretical guidance for the task and function of operational entities in the war [4], [5]. However, when the unforeseeable circumstances appear in the war, the land combat individuals may not be able to complete the specified task, even cause heavy casualties, if continuing to operate in accordance with the operational doctrines. The combat individuals with self-organizing capabilities can take favorable measures according to changes in the battlefield situation. The rules of engagement for the combat individuals are defined in MABLWS as followed:

1) The rule for moving. Agent determines which direction to move towards according to the penalty function and weights of attributes. MABLWS uses path planning method based on $\mathrm{A}^{*}$ algorithm seen in literature.

2) The rule for attacking. In a given range $R_{T}$, if the number of friendly individuals is more than $N_{f}$, then attack, otherwise not attack. In other words, if the combat agent can get enough fire support, it will take the offensive strategy.

3) The rule for aggregating. In a given range $R_{c}$, if the number of friendly individuals is less than $N_{f}$, the combat agent will aggregate to other Agents, or else do nothing.

4) The rule for firing. Individuals take different firing rules based on attribute weights to fight enemies in the shooting range. If it is more aggressive, it will choose any individual in the shooting range to attack. If it is hitted, it will be injured or destroyed.

5) The rule for chasing. When agent senses that the number of opposing agents around it is less than a specific threshold $N_{e}$, it will attack those without regard for its own attribute weights.

6) The rule for retreating. When agent senses that the number of friendly agents around it is less than a specific threshold $N_{f}$, it will retreat.

\section{Path Planning for Ground Simulation Object Based on A* Algorithm[6]}

A* Algorithm is the most effective method of shortest path solution. The heuristic function $F(X)$ introduced, it can be represented by

$$
F(X)=H(X)+G(X)
$$

where $X$ is next point, $G(X)$ is the path dissipation from initial point to next point, and $H(X)$ is the path dissipation estimation of the minimum dissipation path form $X$ to goal point. Accordingly, $F(X)$ is the path dissipation estimation of the minimum dissipation path through $X$. On the supposition that that $H^{*}(X)$ is the path dissipation true value of the minimum dissipation path form $X$ to goal point, it must satisfy

$$
H(X) \leqslant H^{*}(X)
$$

Under this condition, $A^{*}$ Algorithm is adoptable when all path dissipations are more than zero. As a result, the minimum dissipation path form initial point to goal point must be found, and the path is the optimal path.

After the right heuristic function $F(X)$ is selected, $A^{*}$ Algorithm need maintain two tables, called open table and closed table. Closed table records the nodes that has been visited, open table records the nodes that need be searched, and a treelike data structure is used to store the nodes. The nodes in open table are ranked descending order by value of $F(X)$. Fig. 2 is the flow chart of $A^{*}$ Algorithm.

Let $\mathrm{A}$ and $\mathrm{B}$ are the any two points connected directly, $\mathrm{AB}$ is the segment of between A and B. It hasn't other point to be considered between $\mathrm{A}$ and $\mathrm{B}$, that is to say $\mathrm{AB}$ is not intersect with other routes. Before constructing the heuristic function $F(X)$, the defines is given as follows $t(\mathrm{AB})$ traversability index of terrain between $\mathrm{A}$ and $\mathrm{B}, t(\mathrm{AB}) \in$ $[1,0]$, the $t(\mathrm{AB})$ is more smaller, the traversability is more 
worse, $t(\mathrm{AB})=0$ represents that $\mathrm{AB}$ is not passable.

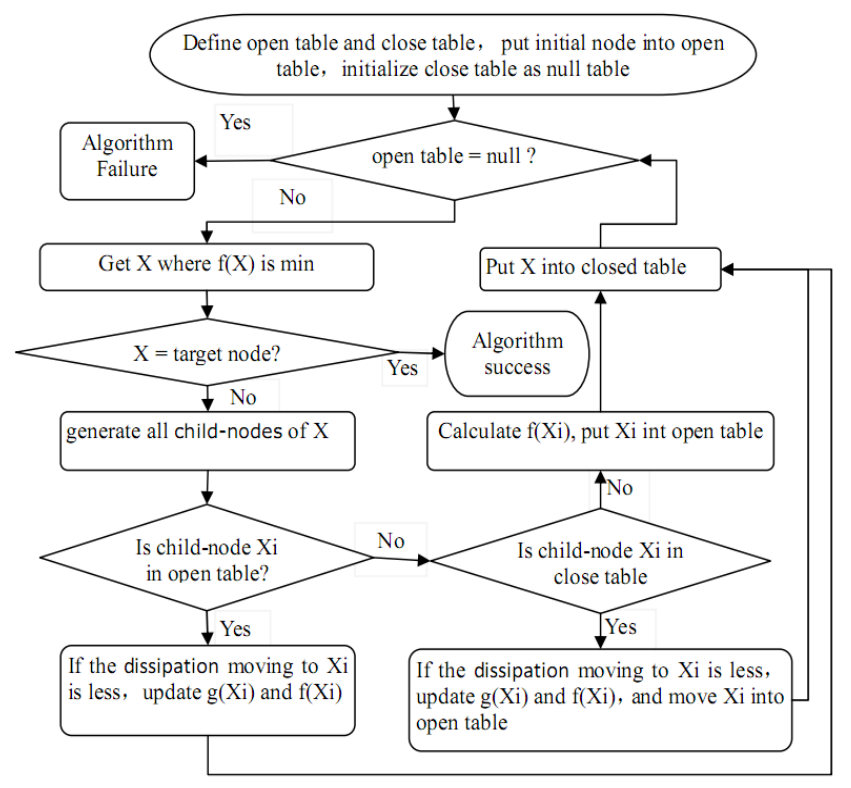

Fig. 2. Flow chart of A* Algorithm.

$S(\mathrm{AB})$ : security index, between $\mathrm{A}$ and $\mathrm{B}, S(\mathrm{AB}) \in[1,0]$, the $\mathrm{S}(\mathrm{AB})$ is more smaller, the security is more worse, $\mathrm{S}(\mathrm{AB})=0$ represents that $\mathrm{AB}$ is the most dangerous.

$C(\mathrm{AB})$ : the dissipation from $\mathrm{A}$ to $\mathrm{B}$, obviously $C(\mathrm{AB})=$ $V(\mathrm{AB})$. In order to consider comprehensively the traversability of terrain and security, $C(\mathrm{AB})$ is described as follow

$$
C(A B)=\left\{\begin{array}{cl}
\frac{|\mathrm{AB}|}{t(\mathrm{AB})^{\alpha} S(\mathrm{AB})^{\beta}} & \text { when } t(\mathrm{AB}) \neq 0 \text { and } S(\mathrm{AB}) \neq 0 \\
\infty & \text { when } t(\mathrm{AB})=0 \text { and } S(\mathrm{AB})=0
\end{array}\right.
$$

where $\alpha$ and $\beta$ are the weight constant. When moving along $\mathrm{AB}$, The define above expresses that the dissipation and the distance between $A$ and $B$ are in direct proportion. When $t(\mathrm{AB})=0$ and $S(\mathrm{AB})=0$, the dissipation is $+\infty$, therefore the optimal path will exclude $\mathrm{AB}$. The parameter $\alpha$ and $\beta$ is used to control the effect of traversability and security on the value of $C(\mathrm{AB})$. if $\alpha>\beta$, the effect of $C(\mathrm{AB})$ on $t(\mathrm{AB})$ is much lower than the effect of $C(\mathrm{AB})$ on $S(\mathrm{AB})$, the optimal path is emphasis particularly on security; if $\alpha<\beta$, the effect of $t(\mathrm{AB})$ on $C(\mathrm{AB})$ is much lower than the effect of $S(\mathrm{AB})$ on $C(\mathrm{AB})$, the optimal path is emphasis particularly on traversability. If the security is not considered, $1=\alpha$ and $0=$ $\beta$ are set.

Let $S_{0}$ denote the initial point, $D_{0}$ denote the goal point, $\Omega_{0}=S_{0} A_{1} A_{2} A_{n}$ denote any folding line on the map, $g\left(A_{n}\right)$ denote the dissipation passing through $\Omega_{0}$ to $A_{n}$. Because the heuristic function satisfies $(1), g\left(A_{n}\right)$ can be represented by

$$
g\left(A_{n}\right)=c\left(S_{0} A_{1}\right)+\sum_{1}^{n} c\left(A_{i} A_{i+1}\right)
$$

In order to construct the heuristic function, $H(X)$ need be defined. Let $H\left(A_{n}\right)$ denote the function of the path dissipation estimation form $A_{\mathrm{n}}$ to $D_{0} . H\left(A_{n}\right)$ can be represented by

$$
\mathrm{H}\left(A_{n}\left|A_{n} D_{0}\right|\right) \triangleq
$$

It is necessary to proof the definition to satisfy (2), here $A^{*}$ Algorithm can find the optimal path. Suppose that $\Omega_{1}=$ $A_{n} A_{n+1} \cdots A_{n+k} D_{0}$ is the shortest path from $A_{n}$ to $\mathrm{D}_{0}$, and then

$$
\begin{aligned}
& H^{*}\left(A_{n}\right)=c\left(A_{n} A_{n+1}\right)+\cdots+c\left(A_{n+k} D_{0}\right) \\
& =\frac{\left|A_{n} A_{n+1}\right|}{t\left(A_{n} A_{n+1}\right)^{\alpha} \times s\left(A_{n} A_{n+1}\right)^{\beta}}+\cdots \\
& +\frac{\left|A_{n+k} D_{0}\right|}{t\left(A_{n+k} D_{0}\right)^{\alpha} \times s\left(A_{n+k} D_{0}\right)^{\beta}} \geq \\
& \left|A_{n} A_{n+1}\right|+\cdots\left|A_{n+k} D_{0}\right| \geq\left|A_{n} D_{0}\right|=H\left(A_{0}\right)
\end{aligned}
$$

By the before-mentioned deduction, the $A^{*}$ Algorithm is adoptable, here the heuristic function can be constructed by the (1).

\section{IMPLEMENTATION OF LAND WARFARE SIMULATION MODELS}

\section{A. Object-Oriented Implementation Framework}

From the relationship between the individual and the environment, the environment should also be a certain viable individual [7], [8]. Therefore, the environment is also a specific agent in MABLWS. According to the objectoriented design pattern, we design and implement the MABLWS framework, as shown in Fig. 3.

Class Agent Sim is the entry point of the simulation, which control to start, pause or end the simulation, and is responsible for initializing both sides. Class Agent Sim Env is operational region for simulation running, which exhibit the process of simulation using two-dimensional grid. There are three parts in class Agent Sim Env: Terrain Manager, Mission Manager and Agent Manager. The terrain manager is responsible to create different types of terrain and show that the type of terrain with the corresponding color; the task manager is responsible for the control operational task execution; the agent manager is responsible for creating, maintaining and destructing agents. Any agent contains a list of rules. Class Agent Manager can provide LOS calculator to support sensing for agent.

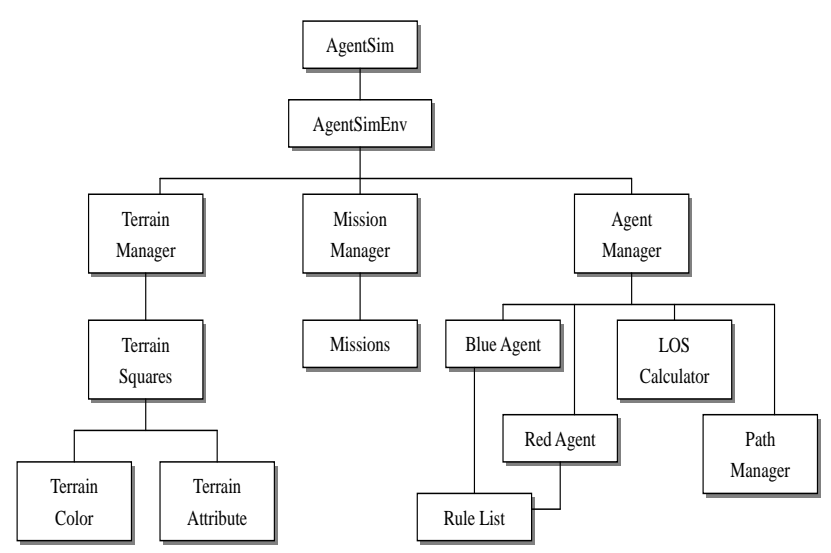

Fig. 3. Object-oriented framework of MABLWS.

\section{B. Establishing the Land Warfare Agent Model}

In the military system, there is a hierarchical relationship between the agents, that is, any agent can internally include the other sub-agent. Agent can concurrently communicate, coordinate and handle the information received. Fig. 4 
illustrates the land warfare agent model and the class diagram of Agent is shown by Fig. 5.

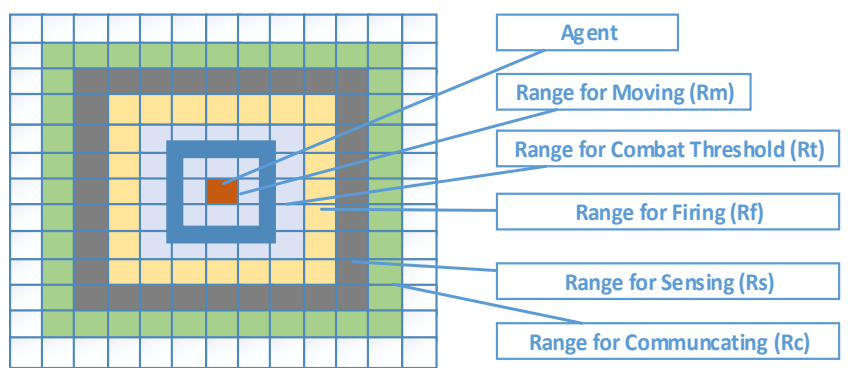

Fig. 4. Illustration of the land warfare agent model.

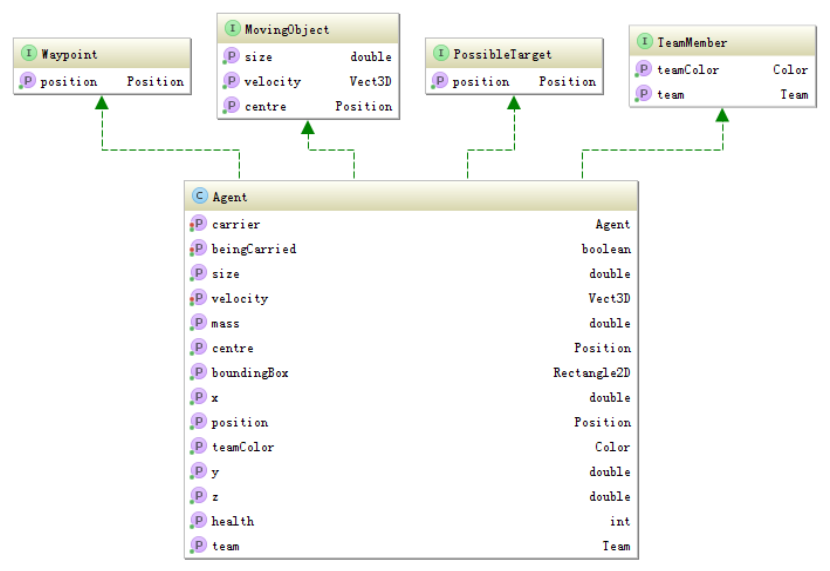

Fig. 5. Class diagram of the land warfare agent model.

The simulation process of MABLWS running in a typical scenario is shown by Fig. 6 .
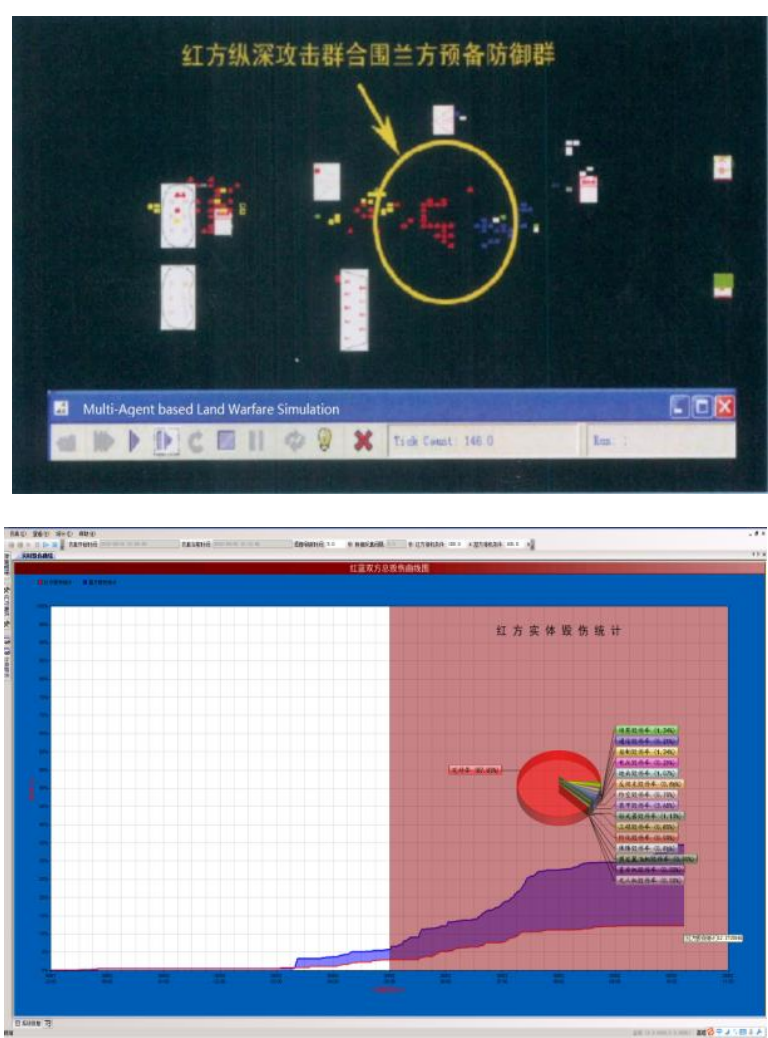

Fig. 6. The screenshots of the simulation process of MABLWS.

\section{CONLUSIONS}

Research on modeling and implementation of the land warfare simulation based on multi-agent method is elaborated in this paper. Multi-agent based land warfare simulation can manifest "emergence" in the macro-level of the complex system. In order to promoting MABLWS more practical, the rules of engagement in more detail will be further investigated on.

\section{ACKNOWLEDGMENT}

The authors of this paper would like to thank Professor Tang Xuemei, Executive Director for Operational Research Societies, who is long engaged in researching on army equipment modeling and simulation. The authors of this paper would also like to thank both Zhao Zhiqiang, Ph.D, and Jiang Bochuan, Ph.D for their encouraging support of the path planning method for the ground simulation object based on $\mathrm{A}^{*}$ algorithm.

\section{REFERENCES}

[1] A. Ilachinski, Land warfare and complexity Part I, Center for Naval Analyses Memorandum CIM-461, 1996.

[2] A. Ilachinski, Land warfare and complexity Part II, Center for Naval Analyses Memorandum CIM-68, 1996.

[3] D. Y. Liu, K. Yang, and J. Z. Chen. "Agents: Present status and trends," Journal of Software, vol. 3, pp. 315-321, Dec. 2000.

[4] Y. Chi and Y. J. Tan. "Study of multi-agent based combat simulation model framework," Computer Simulation, vol. 21, pp. 13-18, April 2004.

[5] D. G. He and Z. L. Wu. "Operational effectiveness analysis of EINSTein system for self-adaptability command and control," Fire Control \& Command Control, vol. 38, pp. 87-90, Jan. 2013.

[6] Z. Q. Zhao, Z. H. Liu, and J X Hao. "Path planning for ground simulation object based on $\mathrm{A}^{*}$ algorithm," Applied Mechanics and Materials, vol. 229-231, pp. 2019-2024, 2012.

[7] F. Y. Liu, G. N. Qi, and H. A. Che, "Research on algorithm for detecting simple path in complex network and its application," Systems Engineering-Theory \& Pracitice, vol. 4, pp. 31-36, May 2006.

[8] Z. L. Dong, D. Jiang, P. Luo, and G. Y Si, "Study on application of complex adaptive system theory in modeling and simulation of war system," Journal of The Academy of Equipment Command \& Technology, vol.17, pp. 5-9, Oct. 2006.

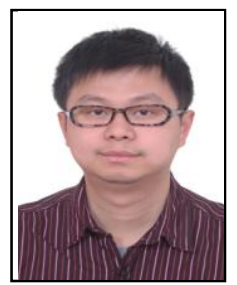

Gan Bin is a system engineer with the Science and Technology on Complex Systems Simulation Laboratory (SSL) at Beijing. He is working a number of research efforts in application of MAS-based and HLA-based technologies to military modeling and simulation. He has been involved with various system integration projects, and is responsible for the distributed simulation integration and demonstrations in the army. He earned M.S. Degree in National University of Defense Technology.

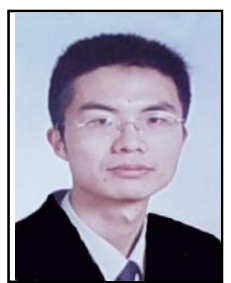

Hao Jiaxin is a software engineer with the Science and Technology on Complex Systems Simulation Laboratory (SSL) at Beijing. He has a rigorous understanding of Linux/Unix development with experience in $\mathrm{C} / \mathrm{C}++$, LISP, Assembly, OpenGL, Gtk, X-windows, Gnome, and the POSIX system libraries. He also has experience with MS Windows development using their Win32 API, MFC libraries, and DirectDraw/DirectSound SDKs. Mr. Hao received his M.S. Degree from National University of Defense Technology on 2006. 